\title{
Advances in Targeted Re-innervation
}

\section{Ali Izadpanah*}

Division of Plastic and Reconstructive Surgery, McGill University Health Centre, Canada

*Corresponding author: Izadpanah A, Division of Plastic and Reconstructive Surgery, McGill University Health Centre, Canada, E-mail: ali.izadpanah@gmail.com

Received date: December 24, 2015; Accepted date: December 30, 2015; Published date: January 6, 2016

Copyright: () 2016, Izadpanah A. This is an open-access article distributed under the terms of the Creative Commons Attribution License, which permits unrestricted use, distribution, and reproduction in any medium, provided the original author and source are credited.

\section{Editorial}

In recent years there have been numerous advances in the field of prosthetics. Targeted reinnervation is a newly developed surgical procedure used to improve the motor control and sensation of the affected limb [1-6]. In this procedure, the residual nerves from the amputated extremity are identified and transferred to new "targets" that have otherwise lost their function [1]. These newly innervated muscles and targets are then used as amplifiers for the amputated nerves in order to restore the functional loss. Use of these newly innervated muscles or at times even only parts of muscles (two heads of pectoralis after a forequarter amputation) would allow a more innate control of the bio-prosthetics.

This will not only allow the new muscle contractions to be sensed and used to control prosthesis, it will also enable the overlying skin to have protective sensation. In addition, this will allow the possibility of innervating muscles to have biofeedback and for the patient to simply "think" of the function in order to more easily rehabilitate to the lost function of the limb. For an example, dorsiflexion of foot and the information being travelled by common peroneal nerve will stimulate the prosthetics' sensors placed on the targeted re-innervated muscles so that the patient does not need to relearn new muscle activations according to the remaining muscles to be picked up and activate the prosthesis. This will make the rehabilitation and the use of prostheses much easier and cause the patients to have much higher compliance to the prosthesis. Interestingly, new recent studies have shown a substantial decrease in the neuroma pain associated with amputated part after targeted re-innervation, even more effective than nerve implantation.

Numerous new advances have been made in this field for restoring the sense of touch [7]. New prostheses are being developed to provide a sense of touch to the bio-prosthetics limb. This would revolutionize this field and allow many patients with limited options for restoring function to have near normal life-experiences.

In conclusion, with advances in technology and new avenues of nerve regeneration, I foresee a day that composite vascularized tissue allograft (limb transplant) with its inherent need for highly toxic immunosuppressants to be completely replaced by high-functioning bio-prosthetics with lower cost and required medical/surgical expertise. I encourage the reader to have eyes open for many more advances to be expected in this field in near future.

\section{References}

1. Kuiken $\mathrm{T}$ (2006) Targeted reinnervation for improved prosthetic function. Phys Med Rehabil Clin N Am 17: 1-13.

2. Kuiken T (2005) Prosthetic command signals following targeted hyperreinnervation nerve transfer surgery. Conf Proc IEEE, Eng Med Biol Soc 7: 7652-7655.

3. Kuiken TA (2004) The use of targeted muscle reinnervation for improved myoelectric prosthesis control in a bilateral shoulder disarticulation amputee. Prosthet Orthot Int 28: 245-253.

4. Kuiken TA (2009) Targeted muscle reinnervation for real-time myoelectric control of multifunction artificial arms. JAMA. 301: 619-628.

5. Kuiken TA (2007) Redirection of cutaneous sensation from the hand to the chest skin of human amputees with targeted reinnervation. Proc Natl Acad Sci 104: 20061-20066.

6. Kuiken TA (2007) Targeted reinnervation for enhanced prosthetic arm function in a woman with a proximal amputation: a case study. Lancet. 369: 371-380.

7. Yao J (2011) Sensory cortical re-mapping following upper-limb amputation and subsequent targeted reinnervation: a case report. Conf Proc IEEE Eng Med Biol Soc 1065-1068. 